\title{
Microwave Group Delay Time Adjuster Using Parallel Resonator
}

\author{
Sangkeun Park, Student Member, IEEE, Heungjae Choi, Student Member, IEEE, and Yongchae Jeong, Member, IEEE
}

\begin{abstract}
This letter describes the design of a group delay time adjuster (GDTA) using a parallel resonator. The GDTA consists of a variable capacitor and a variable equivalent inductor. These components are controlled by two bias voltages separately. The variable equivalent inductor is realized using a high impedance transmission line terminated with the variable capacitor. Group delay time can be adjusted by varying the capacitance and the inductance while keeping the fixed resonance frequency. When the proposed GDTA is fabricated on the Korean RFID frequency band (908.5-914 MHz), we could obtain about 3 ns group delay time variation with excellent flatness.
\end{abstract}

Index Terms-Group delay, resonance circuit, varactor diode, variable inductor.

\section{INTRODUCTION}

A SYSTEM performance is limited due to the nonlinearity. The nonlinearity of a system can be explained as AM-to-AM, AM-to-PM, intermodulation distortion, and adjacent channel power ratio, etc. Several linearizing techniques have been introduced to overcome these nonlinearities [1], [2]. When a predistortion or a feedforward technique is applied to the nonlinear system, group delay time matching as well as amplitude and out-of-phase matching are very important. A variable attenuator and a phase shifter are widely used for the magnitude and the phase control [3], [4].

Moreover, a feedback interference signal originated from transmitter (Tx) antenna of the same site deteriorates the performance of receiver ( $\mathrm{Rx})$ system and result in the co-channel interference in the repeating system. The delay time of the co-channel interferer from $\mathrm{Tx}$ to $\mathrm{Rx}$ is different case by case and due to the environmental condition. The amplitude, phase, and electrical delay time of the correction signal are adjusted to cancel the broadband interferer effectively [5], [6].

Until now there have been few GDTA in microwave circuits. The GDTA which consists of different paths having different physical length was introduced [7]. However, the previous GDTA could not control the group delay time adaptively. In this letter, the GDTA which controls group delay time is proposed. The proposed GDTA can play a key role in a number of applications which require group delay time compensation for a proper group delay matching.

Manuscript received May 3, 2006; revised November 1, 2006. This work was supported by the CBNU funds for Overseas Research under Grant OR-2006-4.

The authors are with the Department of Information and Communication Engineering, Chonbuk National University, Jeonju 561-756, Korea (e-mail: cutop@chonbuk.ac.kr).

Color versions of one or more of the figures in this paper are available online at http://ieeexplore.ieee.org.

Digital Object Identifier 10.1109/LMWC.2006.890331

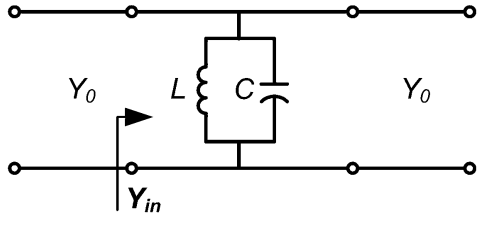

Fig. 1. Shunt resonance circuit.

\section{AdJUSTABle GROUP DELAY THEORY}

A group delay gives the measure of how long it takes to traverse a system. In general, the changing rate of the total phase shift with respect to angular frequency is called the group delay (G.D.), defined as [8]

$$
\text { G.D. }=-\frac{d \varphi}{d \omega}
$$

where $\varphi$ and $\omega$ denote the total phase shift and the angular frequency, respectively. Also, the group delay flatness in the operating frequency band is an important parameter for observing phase linearity of a receiver system, transmitted signal, and so on.

We need to analyze a resonant circuit shown in Fig. 1. The input admittance of the resonant circuit is expressed as (2) and the transmission characteristic can be expressed as (3)

$$
\begin{aligned}
Y_{\mathrm{in}}= & Y_{0}+j\left(\omega C-\frac{1}{\omega L}\right) \\
S_{21}= & \frac{2 Y_{0}}{\sqrt{4 Y_{0}^{2}+(\omega C-1 / \omega L)^{2}}} \\
& \times \exp \left(j\left(\tan ^{-1} \frac{1-\omega^{2} L C}{2 \omega L Y_{0}}\right)\right) .
\end{aligned}
$$

We derived (4), the differential phase component of transmission coefficient with respect to angular frequency, from (3). If we maintain the particular resonance frequency, $\omega_{0}^{2} L C=1$, of the parallel resonator, the magnitude, and the phase coefficient would be maintained constantly. However, the group delay time can be expressed as

$$
\text { G.D. }=\frac{2 Y_{0} L\left(1+\omega^{2} L C\right)}{4 \omega^{2} L^{2} Y_{0}^{2}+\left(1-\omega^{2} L C\right)}=\frac{1}{\omega_{0}^{2} Y_{0} L}=C Z_{0} .
$$

From (4), the group delay time increases proportional to the capacitance. On the contrary, as the inductance increases, group delay time decreases, proving the inverse proportionality to the inductance. Keeping the fixed resonant frequency, the group delay time can be adjusted by the several combinations of a capacitance and an inductance. 


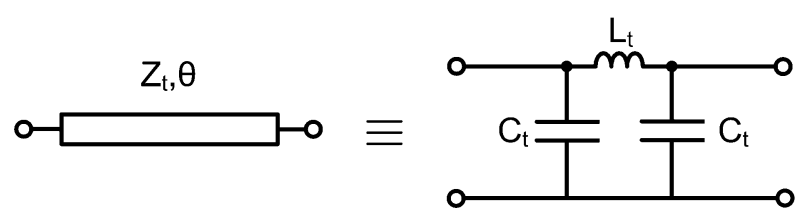

Fig. 2. Transmission line and a lumped element equivalent circuit.

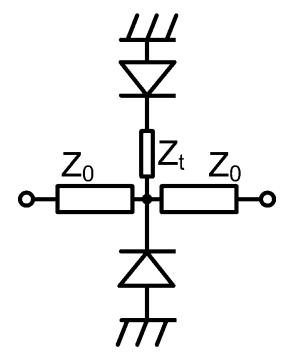

Fig. 3. Proposed GDTA unit.

\section{IMPLEMENTATION AND MEASUREMENT OF THE GDTA}

\section{A. Varactor Diode Measurement}

A varactor diode is a semiconductor device that is widely used in many applications where we require a variable capacitance. The operation of the varactor diode is based on the fact that a reverse biased $\mathrm{PN}$ junction acts as a variable capacitor. The used diode capacitance of 1T362 of Sony versus reverse voltage has a variation of about 2.3 to $100 \mathrm{pF}$.

\section{B. A Variable Equivalent Inductor and the GDTA Unit}

There are few variable inductors in microwave devices. Even though there is an active inductor using a gyrator structure that can change an inductance, a quality factor ( $Q$-factor) is not high enough and is changed according to the control voltage [9]. For that reason, the active inductor is not yet widely used. The series combination of lumped inductor and varactor diode can be used as a variable equivalent inductor. However, since it is difficult to fabricate high $Q$ inductors with small tolerance, the combination of varactor diode and lumped inductor is not suitable. A transmission line terminated with the varactor can be also used as the variable inductor. However, the physical length of transmission line is too long in case of the low operating frequency.

In this work, a high impedance transmission line terminated with the varactor is used to implement the variable inductor. Fig. 2 shows the lumped element equivalent circuit of the transmission line. $Z_{t}$ and $\theta$ are characteristic impedance and electrical length of the transmission line, respectively. The values of the equivalent lumped elements are represented as

$$
L_{t}=\frac{Z_{t} \sin \theta}{\omega}, \quad C_{t}=\frac{1-\cos \theta}{Z_{t} \omega \sin \theta} .
$$

Using the varactor diode and the proposed variable equivalent inductor, we design the GDTA unit shown in Fig. 3. The varactor diode is operated as the variable capacitance, and the transmission line terminated with the varactor diode is operated as the variable equivalent inductor.

The transformation procedure of the variable equivalent inductor is depicted at Fig. 4. Capacitor $C_{1}$ denotes the variable

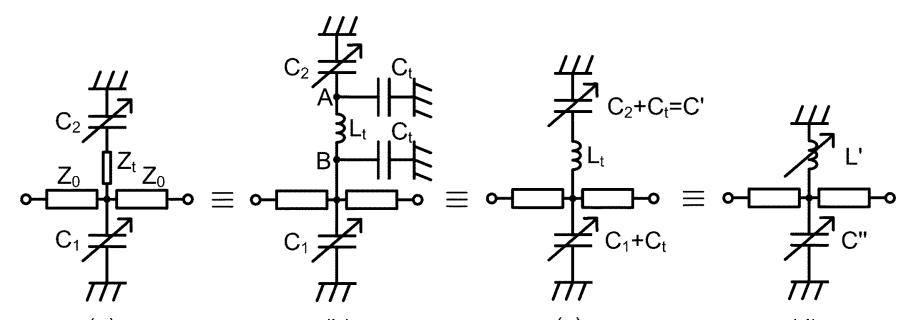

(a)

(b)

(c)

(d)

Fig. 4. Equivalent circuit of the GDTA using the transmission line.

TABLE I

GDTA UNIT MEASUREMENT (AT $911 \mathrm{MHz}$ )

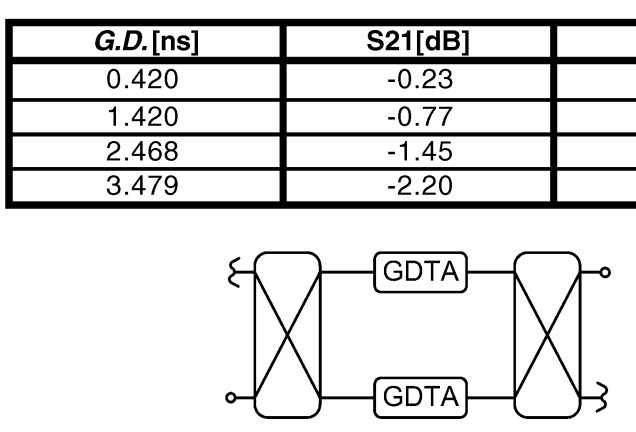

Fig. 5. Block of the proposed balanced GDTA.

capacitance, and $C_{2}$ is used for the variable equivalent inductor with the high impedance transmission line, respectively. We replaced high impedance transmission line with lumped element equivalent circuit as shown in Fig. 4(a) and (b). Since $C_{2}$ shares node A with $C_{t}$, and $C_{1}$ shares node B with $C_{t}$, we can substitute those pairs of capacitors with $C^{\prime}$ and $C_{1}+C_{t}$, as shown in Fig. 4(c). Finally, $C_{1}+C_{t}$ can be represented as $C^{\prime \prime}$, and series connection of $L_{t}$ and $C^{\prime}$ can be substituted with $L^{\prime}$. Equation (6) shows the equivalent reactance of the transmission line terminated with the varactor diode. As long as the equivalent reactance $\left(X_{L}\right)$ is positive, it has an inductive characteristic. Therefore, as $C^{\prime}$ is varied, we can obtain the variable inductance

$$
X_{L}=\frac{\omega_{0}^{2} L_{t} C^{\prime}-1}{\omega_{0} C^{\prime}} .
$$

The value of the variable capacitor and inductor are controlled by two separate bias voltages, and they must satisfy the fixed resonance condition. The measured result of the proposed GDTA unit tested at $911 \mathrm{MHz}$ is shown in Table I.

\section{Balanced GDTA}

In order to obtain better reflection characteristics of the GDTA, a balanced GDTA structure is proposed and shown in Figs. 5 and 6. It is composed of two hybrid couplers (RF Power, S03A888N1) and two GDTA units. The overall circuit size is $79 \times 39[\mathrm{~mm}]$. The implemented GDTA is tested on RFID Korean frequency band (908.5-914 MHz). The group delay measurement of the proposed balanced GDTA is represented at Table II and Fig. 7.

Although we could obtain more group delay time variance more than $3 \mathrm{~ns}$, the transmission and the group delay time flat- 


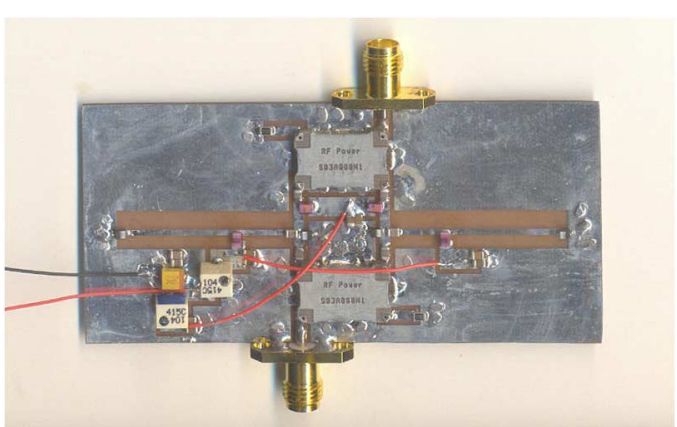

Fig. 6. Fabricated balanced GDTA.

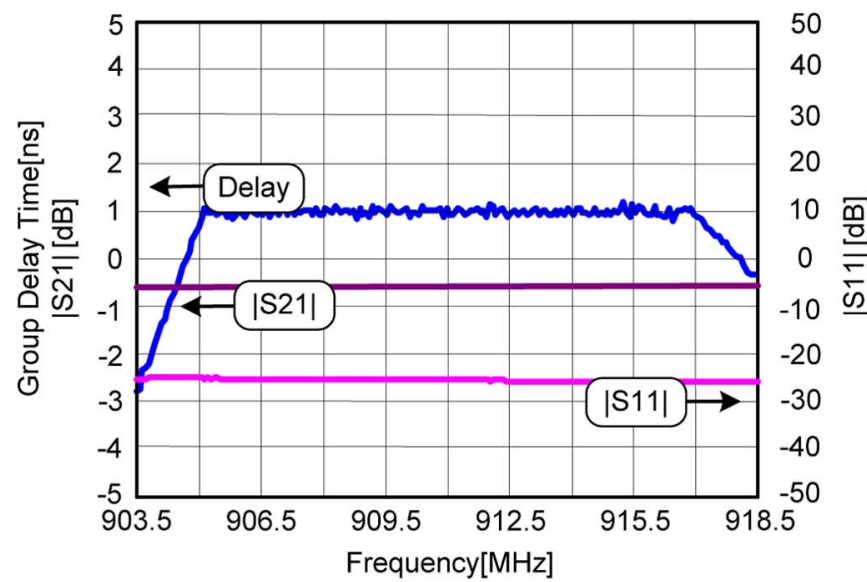

(a)

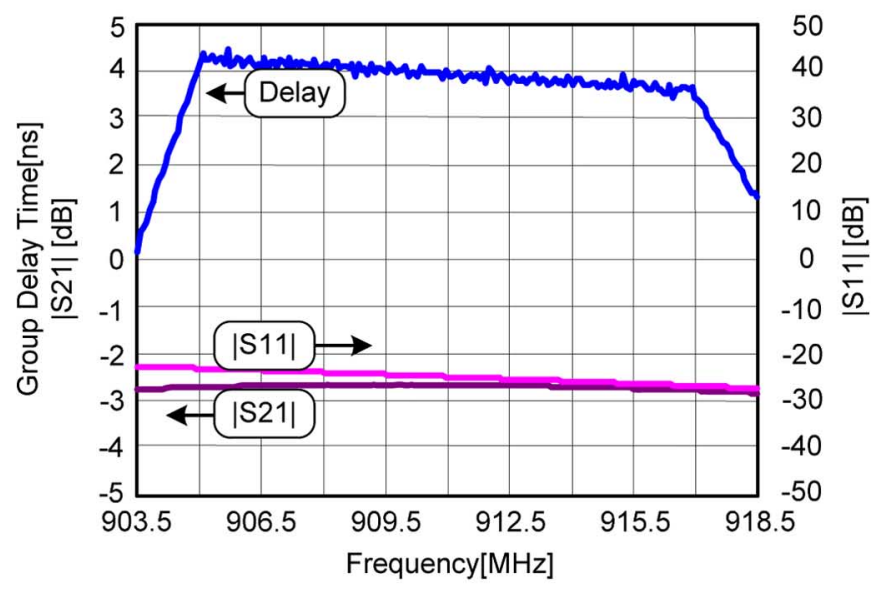

(b)

Fig. 7. Electrical characteristics of the balanced GDTA. (a) Minimum group delay time. (b) Adjustable group delay time.

ness in the high group delay time region are in a trade-off relation so that we have no choice but to limit the actual variation range as $3 \mathrm{~ns}$. In that case, the magnitude flatness is less than $0.1 \mathrm{~dB}$ in the pass band and the maximum reflection coefficient
TABLE II

BALANCED GDTA MEASUREMENT RESUlts

\begin{tabular}{|c|c|c|c|c|c|c|c|c|}
\hline \multicolumn{3}{|c|}{ G.D.[ns] } & \multicolumn{3}{|c|}{$\mathrm{S} 21[\mathrm{~dB}]$} & \multirow{2}{*}{$\begin{array}{l}\text { S11, max } \\
\text { [dB] }\end{array}$} & \multicolumn{2}{|c|}{$\begin{array}{c}\text { Control } \\
\text { Voltage[V] }\end{array}$} \\
\hline $\begin{array}{l}908.5 \\
\mathrm{MHz}\end{array}$ & $\begin{array}{c}911 \\
\mathrm{MHz}\end{array}$ & $\begin{array}{c}914 \\
\mathrm{MHz}\end{array}$ & $\begin{array}{l}908.5 \\
\mathrm{MHz}\end{array}$ & $\begin{array}{c}911 \\
\mathrm{MHz}\end{array}$ & $\begin{array}{c}914 \\
\mathrm{MHz}\end{array}$ & & $\mathbf{v}_{\mathrm{c}}$ & $V_{L}$ \\
\hline 1.005 & 1.041 & 1.025 & -0.65 & -0.64 & -0.64 & -25.65 & 25.0 & 0.0 \\
\hline 2.000 & 2.010 & 1.970 & -1.36 & -1.37 & -1.39 & -26.74 & 10.0 & 14.4 \\
\hline 3.051 & 3.077 & 2.986 & -1.96 & -1.95 & -1.95 & -24.84 & 8.3 & 17.5 \\
\hline 4.021 & 3.938 & 3.792 & -2.68 & -2.68 & -2.71 & -24.41 & 7.0 & 19.8 \\
\hline
\end{tabular}

is about $-24.4 \mathrm{~dB}$, satisfactory results to be applied to the systems where the group delay time matching with good flatness is essential.

\section{CONCLUSION}

We designed a new GDTA unit which can control the group delay time of a signal using the parallel resonant circuit. Keeping the fixed resonance frequency, the group delay time can be adjusted by the combination of values of capacitance and inductance. The fabricated balance GDTA improves the poor reflection characteristic of the single GDTA unit and presents the group delay time variation of about $3 \mathrm{~ns}$. Now we have a plan to apply the GDTA to the wireless communication systems such as the co-channel interference cancellation technique and feedforward linearization. We think that the proposed GDTA will contribute not only to the improvement of the quality of a communication, but to the simplification of the group delay time tuning procedure of a communication system.

\section{REFERENCES}

[1] Y. C. Jeong, "A feedforward power amplifier with loops to reduce $\mathrm{Rx}$ band noise and intermodulation distortion," Microw. J., vol. 45, no. 1, pp. 80-91, Jan. 2002.

[2] Y. C. Jeong, Y. J. Song, I. J. Oh, and C. D. Kim, "A novel adaptive feedforward amplifier using an analog controller," Microw. J., vol. 46, no. 4, pp. 76-85, Apr. 2003.

[3] Y. C. Jeong, "Design of a novel vector modulator," Microw. J., vol. 44, no. 10 , pp. $156-162$, Oct. 2001.

[4] W. T. Kang, I. S. Chang, and M. S. Kang, "Reflection-type low-phaseshift attenuator," IEEE Trans. Microw. Theory Tech., vol. 46, no. 7, pp. 1019-1021, Jul. 1998.

[5] S. J. Kim, J. Y. Lee, J. C. Lee, J. H. Kim, B. Lee, and N. Y. Kim, "Adaptive feedback interference cancellation system (AF-ICS)," in IEEE MTT-S Dig., Jun. 2003, vol. 1, pp. 627-630.

[6] T. O'Sullivan, R. A. York, B. Noren, and P. M. Asbeck, "Adaptive duplexer implemented using single-path and multipath feedforward techniques with BST phase shifters," IEEE Trans. Microw. Theory Tech., vol. 53, no. 1, pp. 106-114, Jan. 2005.

[7] I. Bahl and P. Bhartia, Microwave Solid Circuit Design. New York: Wiley, 1988, pp. 626-659.

[8] D. M. Pozar, Microwave Engineering, 2nd ed. New York: Wiley, 1998.

[9] R. Mukhopadhyay, Y. Park, P. Sen, N. Srirattana, J. S. Lee, S. Nuttinck, A. J. Joseph, J. D. Cressler, and J. Laskar, "Reconfigurable RFICs for frequency-agile VCOs in Si-based technology for multi-standard applications," in IEEE MTT-S Dig., Jun. 2004, vol. 3, pp. 1489-1492. 